\title{
EL IMPACTO AMBIENTAL DEL DESARROLLO DEL TURISMO EN LAS ISLAS DEL ARCHIPIÉLAGO JARDINES DEL REY, CUBA.

\author{
por
}

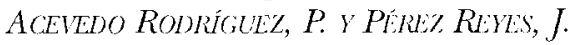 \\ (Facultad de Geografia, Universidad de La Habana, Cuba.)
}

\section{RESUMEN}

El acelerado proceso de desarrollo turístico emprendido en el Archipiélago Jardines del Rey, en la República de Cuba, representa un potencial factor de perturbación ambiental para las pequeñas islas que integran este sistema insular. Estos geosistemas se caracterizan por sus notables valores naturales, alto grado de conservación natural, importantes funciones geoecológicas y particular fragilidad natural; lo que los hace especialmente vulnerables ante los impactos humanos. En el presente trabajo, se caracterizan las islas de este archipiélago y se realiza el análisis del impacto esperado por el desarrollo turístico en las mismas, como una contribución al esclarecimiento de la problemática ambiental futura y para alertar sobre la necesidad de trabajar en la prevención y evaluación de estos problemas, por medio de la planificación ambiental para garantizar así la protección de las islas.

PALABRAS Claves: turismo, islas, medio ambiente, paisajes, impacto ambiental.

\section{ABSTRACT}

The quick process of tourist development undertaken in the Archipelago Jardines del Rey, in the Republic of Cuba, represents a potential factor of environmental interference for the small islands that integrate this insular system; these geosystemes are characterized by their notables natural values, high degree of natural conservation, important geoecologys functions and particular natural fragility, what makes them specially vulnerable before the human impacts. Presently work, the islands of this archipelago are characterized and is carried out the analysis of the impact waited by the tourist development in 
the same ones, like a contribution to the clarification of the problem environmental perspective and to alert about the necessity of working in the prevention and evaluation of these aspects, it stops by means of the environmental planning to guarantee the protection of the islands.

KEY wORDS: tourism, islands, environment, landscape, environment impact.

\section{INTRODUCCIÓN}

La República de Cuba ha decidido en los últimos años, adoptar el fomento del turismo como alternativa para lograr la diversificación y el crecimiento de la economía; renglón que en un futuro próximo constituirá uno de los pilares fundamentales de su desarrollo. En este sentido es propósito del país dirigir los esfuerzos a la inserción de Cuba en el mercado turístico del Caribe con un carácter especial, al promover un tipo de turismo "sano y limpio» en el cual se haga un uso racional de los recursos de interés turístico y se salvaguarde la calidad del ambiente.

Entre los territorios de mayor potencialidad natural para el desarrollo del turismo en Cuba, se encuentran los grupos de islas que bordean la plataforma insular submarina cubana y, en particular, dentro de ellos, el Archipiélago Jardines del Rey, tambiên conocido como "Sabana-Camagüey» donde se localizan importantes recursos de interés para el turismo, asociados a paisajes de alts valor escénico y naturaleza silvestre Más de $250 \mathrm{Km}$. de hermosas playas un entorno marino de grandes riqueza naturales y estéticas, con atractivos for. dos submarinos, ideales para la práctic del turismo de naturaleza y en partici lar de sol y mar.

El turismo, como alternativa de desé rrollo de las islas en la práctica interna cional, ha demostrado que, al mism tiempo que constituye una important fuente de ingresos, resulta también un vía de alteración ambiental, que pued dar lugar con facilidad al deterioro $d$ los recursos y a la pérdida de los valore naturales de las mismas, si se realiza un explotación inadecuada del medio o s abusa del uso de los recursos.

$\mathrm{Al}$ emprender los planes de desarr llo del turismo en el Archipiélago Jardin del Rey, el Estado cubano, a pesar de ] premura inducida por la crítica situ: ción económica del país, se ha propue to desarrollar un modelo de gestiór donde la protección de las islas y defensa de los recursos naturales, fig ren como prioridad indispensable, pas lograr un desarrollo turístico sostenibl integrado armónicamente en la natur leza.

El reto es grande si se tiene en cue ta que la experiencia mundial sobre situación ambiental de las áreas litoral sometidas al uso turístico es gener: mente negativa, con fuerte degradacić 
de los recursos y pérdida de los valores de interés turístico, por procesos como: la erosión de las playas y su contaminación, asociado a la intensa urbanización y las sobrecargas humanas sobre los geocomplejos.

Las islas constituyen geosistemas particularmente especiales del planeta, lo que está dado por su aislamiento natural, la fuerte influencia litoral, el reducido tamaño y poca complejidad geográfica, la baja biodiversidad relativa, el alto endemismo y la fuerte vulnerabilidad a las catástrofes naturales y ambientales, que las hacen muy sensibles al manejo humano.

En general es reconocido que el tamaño, la edad, el origen (génesis), el grado de aislamiento y la ubicación geográfica de las islas, imponen la naturaleza de sus recursos y su susceptibilidad a las alteraciones.

Debido al tamaño, el equilibrio entre población y recursos es más crítico en las islas que en las tierras continentales. Como los límites de las islas son fijos, sus recursos son con frecuencia objeto de sobre-explotación, lo cual determina el agotamiento de bosques, pesquerías, poblaciones faunísticas, aguas y playas. (S. BROOTZ, 1.991)

Las islas pequeñas, jóvenes $\mathrm{y} / \mathrm{o}$ apartadas, suelen tener muy baja biodiversidad y complejidad geográfica y a la vez son muy vulnerables a la degradación del medio ambiente cuando sus recursos se explotan de forma inadecuada. En ellas, las relaciones entre el litoral y la tierra firme son elevadas, de modo que las consecuencias de una mala gestión litoral o de su interior se ven amplificadas en ambos sentidos (S. Brootz., 1991).

Como el número y la extensión de las poblaciones de plantas y animales, sobre todo en las islas pequeñas, es reducido y generalmente con rasgos de endemismo, el riesgo de desaparición de especies importantes por manejo inadecuado es creciente, así como es mayor la vulnerabilidad a la introducción de especies, ante las cuales los individuos autóctonos no han desarrollado mecanismos de defensa para competir con ellas.

Significativamente delicada es la naturaleza de las islas tropicales, donde la dinámica y energía de los procesos geoecológicos son marcadamente activos, desencadenando el rápido deterioro del medio al ser perturbado el equilibrio natural de los geosistemas insulares.

De esta forma, las islas son consideradas como áreas muy sensibles ante las catástrofes naturales y, en general, las condiciones de vida de las poblaciones insulares se hacen extremadamente complejas ante los problemas ambientales de nuestros días. Esta situación generó la convocatoria de la "Primera Conferencia Global sobre Desarrollo Sostenible de los Pequeños Estados Insulares", 
desarrollada en Barbados en 1.994, auspiciada por las Naciones Unidas (O.N.U.), con la finalidad de discutir sus numerosos problemas, buscar unidad y adoptar medidas que permitan mejorar la situación de las naciones islenas ante estos retos.

Con ocasión de este encuentro, el Jefe de Estado de Cuba, Presidente Fidel Castro Ruz manifestaba ante la Conferencia: "Los pobres y los países pequenos tratamos de saber cómo vamos a sobrevivir en las próximas décadas (....) si somos islas a unos cuantos metros sobre el nivel del mar nos preguniamos qué ocurrirá cuando las aguas suban de nivel y si podemos enfrentar las sequías, los ciclones y demás catástrofes climáticas que nos rodean.» (Revista Bohemia, 13-5-1.994). Estas palabras ponen de manifiesto la dramática situación a la que se enfrentan actualmente los pueblos de las pequeñas islas.

\section{EL ARCHIPIÉLAGO JARDINES DEL REY}

El Archipiélago Jardines del Rey, constituye el grupo insular más extenso y numeroso de los subarchipiélagos cubanos integrado por 991 islas o cayos, (NúNEZ JIMÉNEZ, 1.984), (2.517, según VILLEGAS, 1.985), el cual se extiende por la costa norte del centro de la isla de Cuba, desde la península de Hicacos (Matanzas) por el oeste, hasta la Bahía Nuevitas (Camagüey), por el este, a lo largo de $465 \mathrm{Km}$. en dirección noroestesureste, entre los $21^{\circ} 30^{\prime}$ y $23^{\circ} 15^{\prime}$ de latitud norte y $\operatorname{los} 77^{\circ} 00^{\prime}$ y $81^{\circ} 15^{\prime} \mathrm{de}$ longitud oeste, separado de tierra firme por un conjunto de bahías o macrolagu nas de la parte interior de la plataforma insular marina y tiene al norte una de las formaciones coralinas más impor tantes del Caribe.

Las islas de este sistema insular sor reconocibles por sus dimensiones, sóla mente a escala regional, por lo que la: tipologías empleadas para clasificar la islas a nivel planetario, no le son aplica bles. Para éstas, los autores proponer que sean clasificadas como: "islas plata fórmicas acumulativas de barrera", propia de las áreas de las plataformas conti nentales, e insulares submarinas, dond ocurren fuertes procesos acumulativo bajo los efectos marinos: el aporte con tinental y la influencia y control de lo factores geológicos y paleogeográficos ple tafórmicos, que conforman islas sed: mentarias caracterizadas por una edar de formación joven y un aislamient relativo respecto a tierra firme.

En la obra "Cuba, la naturaleza y. hombre», Tomo I, "El Archipiélago" (1.982); el Dr. ANTONIO NúNEZ JIMÉNE divide el grupo insular Jardines del $R_{t}$ en cinco cayerías:

- La más occidental, que se sitúa a cor tinuación de la península d Hicacos, formada por cayos pequr ños. 
- Hacia el este, la cayeria que se extiende desde el cayo Cruz del Padre hasta Fragoso formada por islas mangláricas con un carácter más complicado.

- El tercer grupo denominado de Santa Maria, al noreste de Caibarién, con cayos rocosos y maravillosas playas.

- La cuarta cayería constituída por los cayos interiores extendidos entre Caibarién y Punta Judas de naturaleza calcárea con manifestaciones cársicas y por lo general altos.

- El último grupo conformado a partir de cayo Coco hacia el este, al norte de las provincias de Camagüey y Ciego de Avila y donde se ubican las islas de mayor tamaño como los cayos Romano, Guajaba, Sabinaly otros, formados por llanuras de calcarenitas, con pequeñas alturas dispersas.

Las características físico-geográficas del Archipiélago Jardines del Rey se manifiestan por componentes de la forma siguiente:

- En el relieve, es distintivo la existencia de playas, barras, dunas arenosas, lagunas litorales, escarpes, terrazas marinas y superficies pantanosas. Los cayos presentan una estructura que consiste, generalmente, en superficies marinas acumulativas elevadas, en la porción norte (barlovento), llanuras marinas planas y carsifi- cadas, hacia la parte central y las llanuras bajas pantanosas con manglares hacia el sur (sotavento). Estos relieves se han desarrollado sobre una cobertera sedimentaria de calcarenitas y biocalcarenitas suavemente plegadas y ligeramente dislocadas, y tienen como procesos genéticos fundamentales de formación a los agentes marinos.

- Las condiciones climáticas se caracterizan por presentar un régimen térmico moderado y relativamente estable, con extremos poco acusados y valores medios mensuales que oscilan entre los $25^{\circ} \mathrm{C}$ y $26^{\circ} \mathrm{C}$; así como una alta persistencia de los vientos procedentes del este, con velocidades medias relativamente altas. El comportamiento anual de las precipitaciones demuestra una fuerte estacionalidad del humedecimiento, con la existencia de un período lluvioso, mayo-octubre, con 918,2 $\mathrm{mm}$. y uno seco, noviembre-abril, con $261,9 \mathrm{~mm}$. para un promedio anual de $1.180,1 \mathrm{~mm}$. y una media de 92 días de lluvias al año.

- La hidrología está condicionada por las características del clima, el relieve y la gcología, dando como resultado la infiltración como principal forma de drenaje de las aguas, a través de las arenas y areniscas, para luego escurrir subterráneamente. Esto hace que no exista una red de dre- 
naje manifiesta, en caso de ocurrir elevadas precipitaciones, las aguas corren sin organización de los lugares más altos a los bajos.

- Existen mantos freáticos de agua dulce, de limitado espesor en los cayos mayores, los cuales están sometidos al peligro de la intrusión salina, por su delicado equilibrio con las aguas subterráneas salinas.

- Los suelos son poco desarrollados y poco variados, con un predominio de los procesos incipientes de pedogénesis; aunque se encuentran suelos ferralíticos asociados a las terrazas altas de las islas interiores, al este de Caibarién.

- La flora y la fauna de la región están representadas por especies adaptadas a las condiciones extremas del lugar. El bosque siempre-verde-micrófilo, el matorral xeromorfo costero y subcostero, los complejos de vegetación de costa arenosa y rocosa y los manglares, son las formaciones más representativas. En cuanto a las especies animales, se encuentran insectos, moluscos, anfibios, reptiles, mamíferos y aves, entre otras; incluyéndose algunas especies endémicas y otras de gran importancia que requieren protección. En los cayos grandes se destaca la fauna introducida, como el ganado vacuno y caballar.
En resumen, las características físico. geográficas del territorio están determi. nadas por su insularidad, su localiza. ción intertropical, evolución paleogeo gráfica reciente; el relieve estructuradc por llanuras bajas, de origen marino acumulativo; los complejos litológico: sedimentarios carbonatados, la intensi dad de los procesos hidrodinámico: marinos, el régimen de humedecimien to estacional con tendencia a la seque dad y altas temperaturas, los sistemas di vientos alisios del noreste, la saliniza ción ambiental, poco desarrollo de lo suelos e hidromorfismo parcial; y e xeromorfismo y microfilismo coms rasgo característico de la vegetaciór insular.

Los paisajes actuales de las islas cons tituyen sistemas naturales formados es un proceso evolutivo reciente. En él s originaron los geosistemas actuales qu $\epsilon$ en respuesta a la existencia de condicic nes generales homogéneas de desarrc llo, en conjunción con las particularidá des locales de los procesos geoecológico. hacen distinguible la existencia de $\mathrm{u}$ patrón evolutivo general para el arch piélago, que manifiesta diferencias pa ticulares para las islas, en dependenci de su ubicación en la estructura dina mico-posicional y en el comportamier to de los factores que intervienen en s génesis.

Por ello, existe una clara diferenci ción general del archipiélago en dire 
ción este- oeste con la disminución del tamaño, la complejidad y edad de las islas en este sentido; lo que responde a una desigual manifestación de los factores de formación, principalmente geológicos. De igual forma, aparece una marcada diferencia en el paisaje de las islas en dirección norte-sur, por la influencia de la dinámica océanoatmósfera, en relación con el proceso de formación de las islas. Así se manifiesta una asimetría de las unidades fisico-geográficas entre el borde exterior, barlovento, de barrera y acumulación; y el borde interior, sotavento, acumulativo-pantanoso. Estas regularidades de formación y diferenciación físico-geográfica determinan las características y propiedades principales del paisaje de estas islas.

Los paisajes del Archipiélago Jardines del Rey presentan una organización funcional dinámico -posicional con claras dependencias e interrelaciones entre las unidades del paisaje y dominio de las relaciones horizontales, regidas por la altimetría del relieve y por la acción hidrodinámica del mar; conformando un complejo paisajistico paradinámico de activo intercambio de sustancia y energía, con importante acción de barrera, estructurado por cuatro hileras de funcionamiento dispuestas de forma paralela entre sí, en las que alternan las áreas sumergidas con las emergidas que son:
- La plataforma insular submarina externa.

- El sistema insular.

- La plataforma insular submarina interna.

- La costa de tierra firme.

La hilera dinámico-posicional del sistema insular realiza una importante función de barrera principal en la organización paradinámica regional y en general, se caracteriza por su carácter colector, su situación entre la plataforma insular submarina externa e interna, y las importantes funciones geoecológicas y, particularmente, ecológicas en su carácter de hábitat de valiosas especies de flora y fauna.

Debido a lo anterior, la estructura funcional de Archipiélago Jardines del Rey, hace factible entre los diferentes geosistemas del mismo, la propagación en cadena de los impactos y procesos negativos, que pudieran originarse por los intensos trabajos que el desarrollo del turismo generará en algunos puntos del sistema insular o por el transporte desde tierra firme de estos fenómenos (ACEvedo, P., 1.996).

Las islas de este archipiélago presentan importantes recursos naturales (Acevedo, P., 1.994), en los que se destacan las playas de alta calidad que, en unión a los paisajes de alto valor natural y escénico, el mar y las condiciones climáticas muy favorables, propician un gran potencial para el desarrollo del 
turismo de sol y mar. También son significativos los recursos de carácter biótico que, en general, se encuentran en ecosistemas en buen estado de conservación y tienen un alto valor ecológico, científico y económico. Es de señalar que la distribución de estos recursos no es uniforme y marcadamente se localizan en las islas de mayor complejidad paisajística que presentan la más alta cantidad y variabilidad de recursos naturales.

En general, los paisajes insulares de este archipiélago presentan un buen estado de conservación, donde predominan las condiciones naturales y seminaturales en la mayor parte de las islas y sólo de manera local, se identifican alteraciones significativas de la naturaleza que en los últimos años han sufrido un proceso de rehabilitación natural, de forma tal que los paisajes del Archipiélago Jardines del Rey no presentan actualmente una fuerte transformación antropogénica. La modificación del paisaje está asociada principalmente a las actividades de la tala no controlada en el pasado, la explotación salinera local en algunos cayos y la introducción de especies exóticas de flora y fauna en algunas islas. Ésto está dado fundamentalmente por el carácter despoblado de las islas, lo que es debido a su aislamiento de la isla de Cuba, su reducido tamaño, los limitados recursos naturales (en particular de agua y suelo) y condiciones inhóspitas, dadas por la presen- cia de plagas y vectores; factores que se conjugan con las situaciones históricopolíticas del país, para dar como resultado su actual estado de conservación natural.

El acelerado proceso de asimilación económica que se está efectuando en el sistema insular Jardines del Rey, constituye un fuerte impacto de antropización del paisaje, que producirá intensos cambios en la composición, estructura, funcionamiento y estabilidad de los geosistemas de las islas; así, el desarrollo propuesto para el territorio, originará una fuerte modificación del paisaje, que traerá alteraciones intensas de la naturaleza, por las construcciones de alojamiento, servicios y de apoyo al turismo: los viales, los préstamos de material constructivo (canteras), la expansión de jardines y áreas verdes para la creación de un paisaje cultural adecuado a los requerimientos de la actividad turística diseñada y la presencia humana en las islas. Partiendo de estas premisas, es evidente que el estudio de la situación ambiental y la magnitud del proceso de modificación del paisaje en el archipiélago, se convierten en objetivos fundamentales del planeamiento ambiental de las islas.

El desarrollo del turismo en las islas producirá fuertes impactos que, de forma general, se esquematizan en la Fig. 2, donde se reporta la ocurrencia de situaciones de conflicto como son 
por el uso de suelo entre áreas de utilización para el turismo (1), ubicadas en la cercanía de las playas y las áreas naturales y protegidas del cayo que, de forma permanente, recibirán el impacto por expansión de las primeras (instalaciones de apoyo, jardines, actividades recreativas, tratamiento de residuales, etc.) y el conflicto que se crea en la franja litoral por su uso intensivo para el turismo (2), demandando espacios preferenciales, en busca de las mejores opciones y facilidades para las instalaciones como son: la proximidad a la línea de costa, la ocupación de puntos estratégicos (mejores visuales, accesos, etc.) y la actividad constructiva, que provocarán intensas alteraciones y presiones sobre los paisajes litorales (rellenamientos, construcciones, instalaciones, ocupaciones no autorizadas, etc.).

El área natural del cayo también se verá impactada por la expansión de la red de tráfico vehicular (3), que se genera por las actividades en la isla y, a su vez, existirá una permanente perturbación del ambiente terrestre (4), que producirán los ruidos, el tráfico automotor, la contaminación puntual, el control de plagas, los desbroces y apertura de canteras, la introducción de especies biológicas, incendios accidentales, etc., que presionarán como nuevos elementos de alteración sobre el medio natural.
Sobre la isla y en particular en su entorno natural, también aumentará significativamente la presión humana (5), por personas que con diferentes fines accederán al cayo, contribuyendo a la degradación ambiental del medio como son: los pescadores y cazadores furtivos, los constructores y personal de apoyo o relacionado con los servicios turísticos, los campistas y excursionistas (especialmente los no controlados), las personas atraídas por el contacto con el turismo extranjero, etc., que incidirán sobre el entorno, produciendo un aumento sistemático de su deterioro.

De igual forma, la presión del turismo sobre las áreas litorales de playa puede producir focos de contaminación en éstas y en las aguas litorales (6), por la aparición de desperdicios y otros elementos contaminadores que pueden ser perturbadores en las aguas de las lagunas litorales; situación que requerirá permanente vigilancia y control. También se producirá la inevitable perturbación del ambiente marino (7), por las actividades originadas por el turismo en el mar (pesca, buceo, movimiento de embarcaciones, etc.), que puede ser muy dañina sobre los ecosistemas marinos y en particular en las áreas de arrecifes coralinos.

Especial atención merece la perturbación ambiental que se podrá producir en las macrolagunas interiores (bahías) y la franja de manglar insular, por la 
construcción de los "pedraplenes" de acceso a las islas (8), que constituyen un factor de alto riesgo, como barrera artificial a la circulación natural del sistema.

El desarrollo del turismo en las islas producirá también impactos importantes sobre las áreas de tierra firme; algunos por la competencia y demanda de recursos (9), como el agua y los materiales de construcción y un potente impacto socioeconómico sobre las poblaciones ribereñas (10), que tendrá connotaciones positivas en algunos aspectos como: el empleo, el salario, el aumento de la actividad cultural y de la preparación profesional, etc. Pero también engendrará fenómenos negativos como: la competencia de empleo, inmigraciones masivas, demanda de vivienda y servicios, aumento de albergados y de personal masculino flotante, influencia cultural externa y pérdida de identidad, rechazo al turista y las deformaciones sociales que acompañan al turismo (prostitución, «jineterismo», alcoholismo, drogadicción, etc.), que requieren de un plan especial de atención, que debe insistir en los aspectos educativos de la población, con antelación al desarrollo propuesto, y una amplia participación comunitaria en la solución y toma de decisiones al enfrentar los problemas. Muchos de estos aspectos ya son palpables en la situación ambiental de Cayo Coco, que presenta el más intenso proceso de desarrollo en el archipiélago hasta el momento.

Debe insistirse en que el estudio realizado parte de las consideraciones planteadas en la zonificación territorial realizada para el archipiélago, a partir de los trabajos de planificación diseñados con el propósito del desarrollo armónico del turismo; el cual, adquiere un carácter algo ideal con respecto a la experiencia práctica, en la que seguramente deben ocurrir mayores afectaciones al medio. Como ejemplo de este razonamiento está el hecho de que no se tuvo en cuenta la contaminación ambiental, pues se prevé que ésta sea minimizada en toda el área para las diferentes etapas; sin embargo, en la práctica, ha de ser muy dificil que no se manifiesten focos o problemas de contaminación en el territorio, de lo cual ya existen evidencias, con lo que ya ocurre con el lagoon de cayo Coco, en plena etapa constructiva inicial.

Es por ello, que deben suponerse en realidad, mayores acciones de desestabilización para las islas sometidas a la asimilación turística (incendios, caza furtiva, desbroces no planificados, alteración de proyectos, etc.) que, a su vez, agudicen la vulnerabilidad de los geosistemas, lo que exige extremar las medidas de vigilancia y control de las islas, continuar los estudios de forma sistemática y realizar el monitoreo ambiental para las diferentes etapas de explotación. 
Por todo esto, se hace necesario estudiar con profundidad el proceso de asimilación de estos territorios, para lograr que se conserven sus potencialidades $y$ recursos naturales, mediante la planifica- ción ambiental del desarrollo del turismo en el área, con la inclusión de cargas, usos y medidas de protección adecuadas, que garanticen formas racionales de explotación y conservación natural.

Figura 1. El desarrollo del turismo y su impacto ambiental en las islas del Archipiélago Jardines del Rey. Cuba.

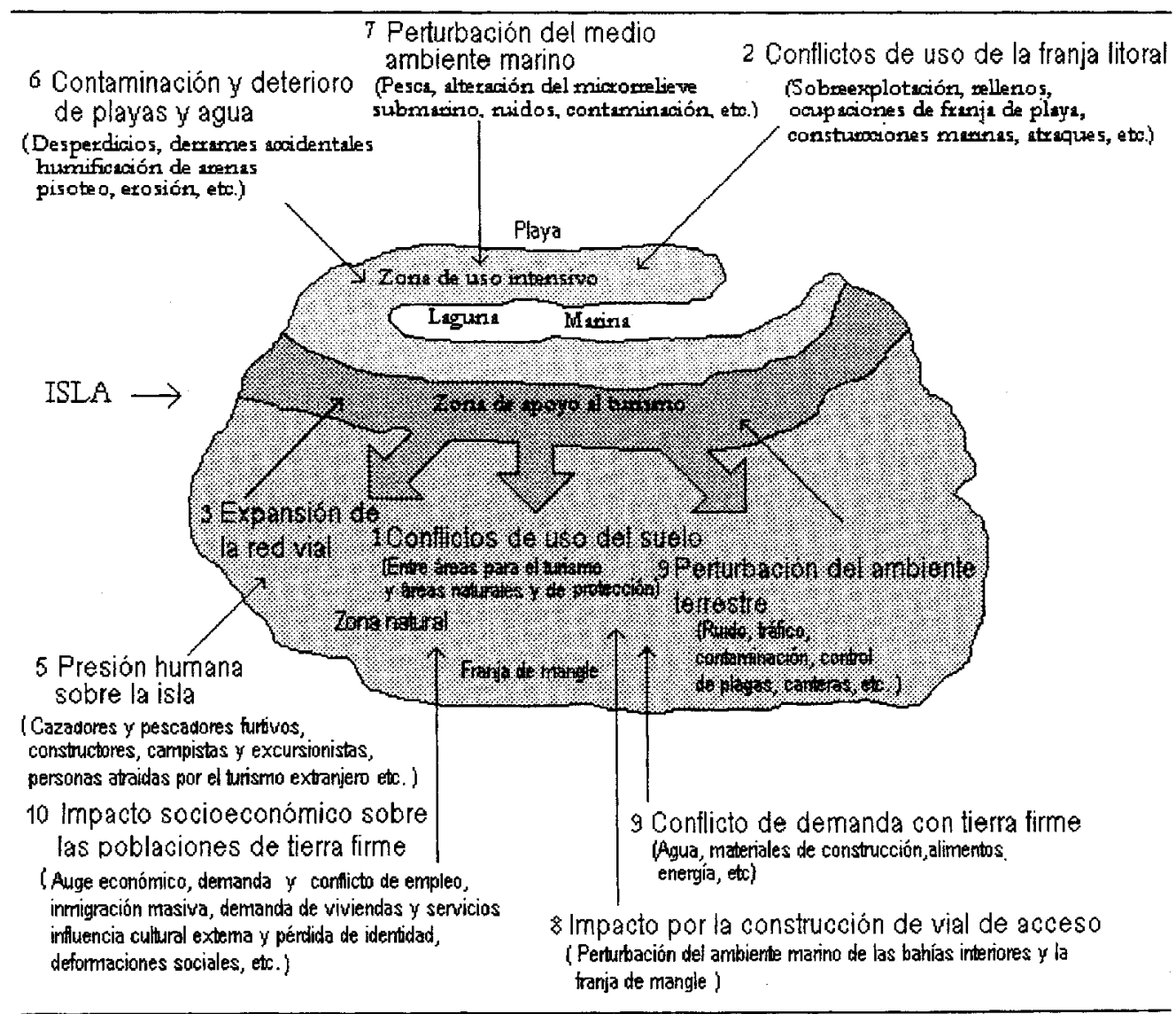

Confeccionado por el autor sobre idea original de E. Gormsen, 1962, para las istas del Caribe. 